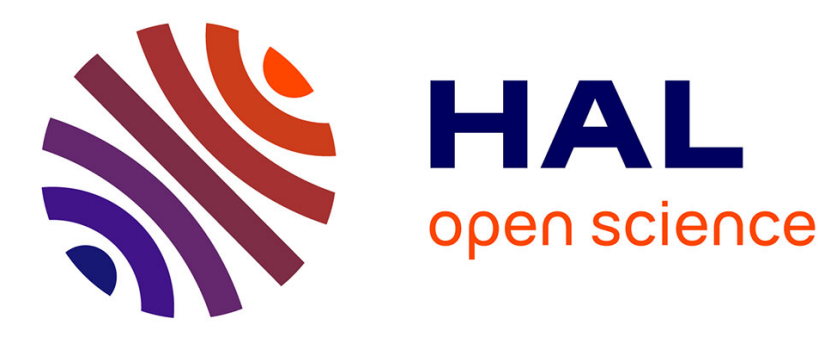

\title{
Fast domain wall dynamics in MnAs/GaAs films
}

M Tortarolo, L. Thevenard, Hans Jürgen von Bardeleben, M Cubukcu, Victor

H. Etgens, M Eddrief, C. Gourdon

\section{To cite this version:}

M Tortarolo, L. Thevenard, Hans Jürgen von Bardeleben, M Cubukcu, Victor H. Etgens, et al.. Fast domain wall dynamics in MnAs/GaAs films. Applied Physics Letters, 2012, 101, pp.072408. 10.1063/1.4746381. hal-01324068

\section{HAL Id: hal-01324068 https://hal.science/hal-01324068}

Submitted on 31 May 2016

HAL is a multi-disciplinary open access archive for the deposit and dissemination of scientific research documents, whether they are published or not. The documents may come from teaching and research institutions in France or abroad, or from public or private research centers.
L'archive ouverte pluridisciplinaire HAL, est destinée au dépôt et à la diffusion de documents scientifiques de niveau recherche, publiés ou non, émanant des établissements d'enseignement et de recherche français ou étrangers, des laboratoires publics ou privés. 


\title{
Fast domain wall dynamics in MnAs/GaAs films
}

\author{
M. Tortarolo ${ }^{1,3}$, L. Thevenard ${ }^{2}$, H. J. von Bardeleben ${ }^{2}$, M. Cubukcu ${ }^{2,4}$, V. Etgens ${ }^{2,3}$, M. Eddrief ${ }^{2,3}$, C. Gourdon ${ }^{2}$ \\ 1 Laboratoire de Chimie Physique, Université Pierre et Marie Curie CNRS UMR 7614, \\ 11 rue Pierre et Marie Curie, F-75005 Paris, France \\ 2 Institut des Nanosciences de Paris, Université Pierre et Marie Curie, \\ CNRS, UMR7588, 4 place Jussieu, F-75005 Paris, France \\ 3 International French Argentinian Nanoscience Laboratory LIFAN \\ 4 Laboratoire Nanostructures et Magnétisme, INAC, CEA-Grenoble, France
}

(Dated: May 31, 2016)

\begin{abstract}
Field-induced domain wall (DW) dynamics was investigated in MnAs/GaAs(100) films by means of longitudinal Kerr microscopy and a field pulse technique. Saw-tooth type domains were observed in the studied temperature range. DW velocities up to $950 \mathrm{~m} \mathrm{~s}^{-1}$ were measured at $200 \mathrm{~K}$ and up to $540 \mathrm{~m} \mathrm{~s}^{-1}$ at $290 \mathrm{~K}$, at the beginning of the nucleation of the non-ferromagnetic $\beta$ phase within the ferromagnetic $\alpha$ phase. Different propagation regimes were observed depending on the magnitude of the Walker field compared to the depinning field, which depends on the progressive nucleation of an unordered $\beta$ phase. The results are interpreted in the framework of the one-dimensional model (1D) for DW propagation.
\end{abstract}

PACS numbers: $75.60 . \mathrm{Ch}, 75.78 . \mathrm{Fg}$

The possibility of storing information in magnetic domains separated by DWs moving along domain tracks $[1,2]$ has stimulated a renewed interest in DW propagation in in-plane magnetized materials for spintronics. Most studies are performed on Permalloy (Py) where high DW velocities (up to $1000 \mathrm{~m} \mathrm{~s}^{-1}$ ) can be achieved $[3,4]$. However it is well known that MnAs is a promising candidate for spintronics applications [5-7] as it can be grown on different semiconductors $[8,9]$. Even though DW dynamics in ferromagnetic films has been intensively investigated during the last decade [4, 10, 11], DW propagation in MnAs was studied only recently. Up to now, studies were limited to the low velocity regime (a few tens of $\mu \mathrm{m} \mathrm{s}^{-1}$ ) observed under application of a magnetic field close to the coercive field where DW propagation is governed by pinning defects [12-14]. In particular, a magnetization reversal study reported on the temperature dependent critical scaling behavior of Barkhausen avalanches [14, 15]. This dependency was ascribed to the decrease of the saturation magnetization with temperature due to the magneto-structural phase coexistence of the ferromagnetic $\alpha \mathrm{MnAs}$ phase and the non-ferromagnetic $\beta$ MnAs phase in the $280-330 \mathrm{~K}$ temperature interval.

Field-induced DW dynamics in the intrinsic regime (above the depinning regime) has not been investigated in MnAs yet. The effect of the $\alpha / \beta$ phase coexistence on DW dynamics in intrinsic regimes therefore remains an open question and particularly relevant to potential applications of MnAs in data storage. In this letter we report on the DW dynamics in MnAs/GaAs over a wide range of magnetic fields and temperature, in the pure $\alpha$ phase, and at the onset of the $\beta$ phase $(\mathrm{T}=290 \mathrm{~K})$. Very high DW velocities, up to $940 \mathrm{~m} \mathrm{~s}^{-1}$, i.e. comparable to those found for Py $[3,4]$ were measured. Strikingly, the maximum velocity at $290 \mathrm{~K}$ is still as high as $540 \mathrm{~m} \mathrm{~s}^{-1}$.
Moreover, the analysis of the velocity curves leads to the identification of different dynamical regimes, depending on the relative values of the field at the end of the depinning regime and the Walker field $\mathrm{H}_{W}$. As depinning critically depends on temperature for MnAs/GaAs due to its characteristic phase coexistence, the DW velocity curve shows different shapes depending on temperature.

The sample is a $300 \mathrm{~nm}$ thick MnAs/GaAs (100) film grown as described elsewhere $[9,16]$, in the epitaxial condition $\operatorname{MnAs}(-1100) \| \operatorname{GaAs}(100)$ with $\operatorname{MnAs}[0001] \| \mathrm{GaAs}[1-10]$. This determines a well-defined uniaxial magnetic anisotropy in the sample plane with the easy axis along the [11-20] direction, an intermediate anisotropy axis along [-1100] and a hard one along [0001]. High resolution magnetic force microscopy measurements at remanence showed that in $\mathrm{MnAs} / \mathrm{GaAs}$ (100) the DWs are $180^{\circ}$ Bloch type [17]. The sample was placed in a helium-flow cryostat. The hysteresis cycles were measured by magneto-optical longitudinal Kerr effect for several temperatures from $200 \mathrm{~K}$ to 290 $\mathrm{K}$, showing square loops in this range of temperature. The temperature dependence of the coercive fields $\mathrm{H}_{c}$ obtained from these loops (Fig. 1, inset) shows a characteristic increase similar to that of Ref. 18. At $200 \mathrm{~K}$, the MnAs film exhibits a single $\alpha$-phase structure. The progressive nucleation of the $\beta$ phase is initiated above $200 \mathrm{~K}$. It remains an unordered phase up to $280-290 \mathrm{~K}$, beyond which it adopts a self-organized stripe pattern, leading to a strong increase of the coercive field [18, 28].

The DW dynamics depends critically on the micromagnetic parameters: magnetization, magnetic anisotropy constants and exchange constant $A$. The magnetization was measured by a superconducting quantum interference device magnetometer. The magnetic anisotropy constants and the exchange constant were obtained from ferromagnetic resonance (FMR) experiments using a Q- 
band setup. The range of available static fields allows the determination of the magnetic anisotropy constants only at $295 \mathrm{~K}$. Taking into account the fraction of the $\alpha$ phase and the demagnetization factor of the $\alpha$ phase stripes [19], we obtain $K_{2 \perp}=3.210^{5} \mathrm{~J} \mathrm{~m}^{-3}$ and $K_{2 \|}=410^{5} \mathrm{~J} \mathrm{~m}^{-3}$ for the $\alpha$-phase using the same definitions as in Ref. [19]. We determine $A$ from the linear fit of the successive spin-wave resonances as a function of the square of the mode number (Fig. 2), assuming Kittel's boundary conditions. We obtain $A=6.6 \mathrm{pJ} \mathrm{m}^{-1}$ at $285 \mathrm{~K}$ [20]. A reliable determination of $A$ at higher temperature is not possible because of additional lines in the spectra.

We then observed the magnetic domains by longitudinal Kerr microscopy, using a LED source $(\lambda=635$ $\mathrm{nm}$ ), a high numerical aperture (0.4) objective and an off-centered aperture diaphragm to probe the horizontal component of the magnetization. The contrast was enough to accurately image the magnetic domains up to $290 \mathrm{~K}$. The field was aligned along the easy axis of the sample. In this configuration we observed wedge shaped domains in the full range of studied temperatures (Fig. 3). The domains expanded along the easy axis direction, much like the saw-tooth domains observed in Refs. [13, 14]. This kind of pattern is known to develop in thin ferromagnetic films with strong uniaxial in-plane anisotropy in order reduce the magnetic charge density when the magnetization of two adjacent domains meets head-on [21]. The DW velocity was then measured using a pulsed magnetic field technique. To be able to track high velocities, pulses were generated by micro-coils placed inside the cryostat, and DW motion was driven by a sequence of field pulses of varying duration. Details can be found in Ref. [22]. The DW velocity was systematically measured at the site of the largest DW displacement, i.e. at the tip of the wedge domains, as indicated by the black line in Fig. 3b. DW displacements were measured in areas with no visible pinning effects.

Figure 1 shows the velocity measurements for several temperatures: 200, 270 and $290 \mathrm{~K}$. The DW velocity becomes measurable above the depinning field $H_{\text {dep }} . H_{\text {dep }}$ is of the order of the coercive field, which explains the unusual increase of the depinning field with the temperature because of the progressive growth of the $\beta$ phase fraction (inset). At high field $(9 \mathrm{mT})$ and $270 \mathrm{~K}$, thermally activated domain nucleation prevents further measurement of the DW displacements. At $290 \mathrm{~K}$ however, nucleation is statistically less likely as the volume fraction of the ferromagnetic phase (90-95\%) is decreased compared to the alpha-phase regime. Points can then be taken up to $14 \mathrm{mT}$ without problems. Above $H_{d e p}$ the velocity rises up to a velocity peak of $900 \mathrm{~m} \mathrm{~s}^{-1}$ at $200 \mathrm{~K}, 480 \mathrm{~m} \mathrm{~s}^{-1}$ at $270 \mathrm{~K}$ and $540 \mathrm{~m} \mathrm{~s}^{-1}$ at $290 \mathrm{~K}$ and then decreases. Note that the maximum velocities are comparable to the velocities measured in Py magnetic tracks [4]. The peak velocity of $540 \mathrm{~m} \mathrm{~s}^{-1}$ observed at $\mathrm{T}=290 \mathrm{~K}$ is over a mil-

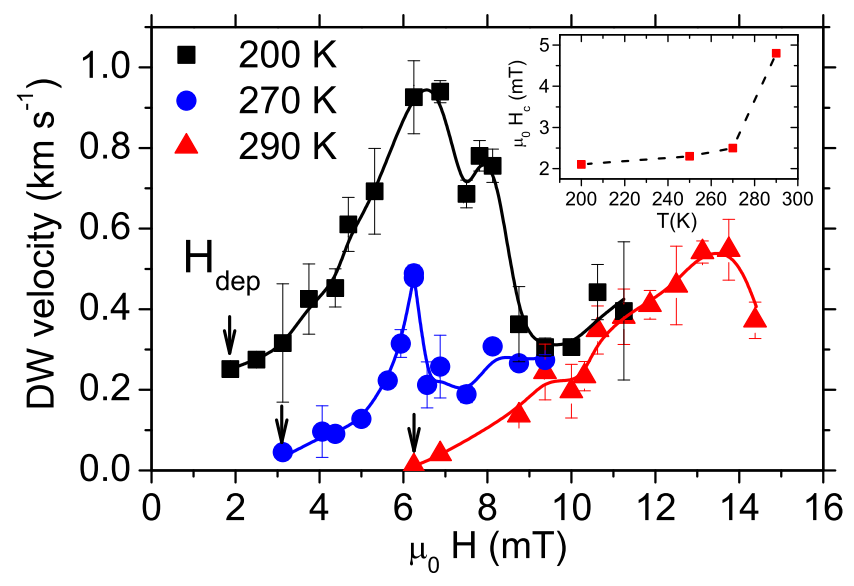

FIG. 1. DW velocity as a function of applied field. The lines are guides for the eyes. (inset) Dependence of the coercive field on temperature.

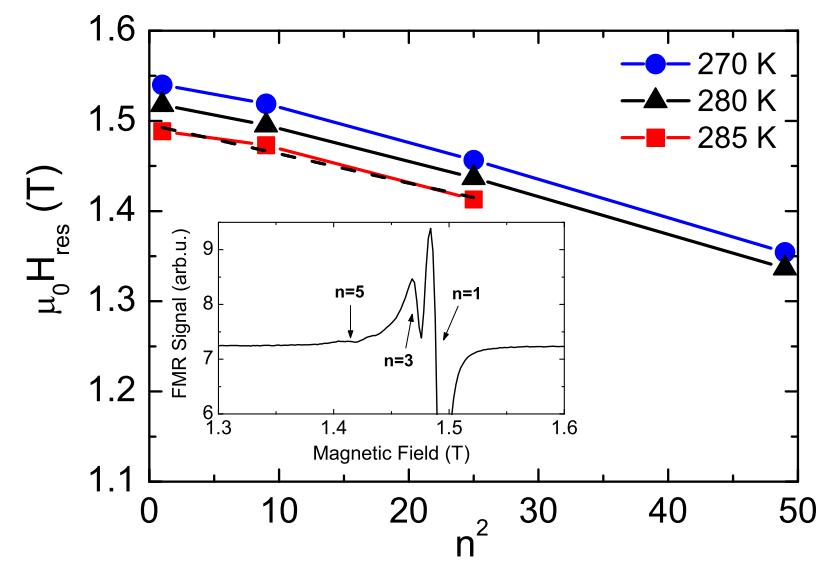

FIG. 2. Dependence of the resonance field of the spin wave modes on the square of the mode number (inset: FMR spectrum at $285 \mathrm{~K}$ ). The dashed line is the linear fit at $285 \mathrm{~K}$ used to determine the exchange constant $A$.

lion fold higher than the velocities that can be deduced from images of magnetization reversal in the coexistence regime previously reported in Ref. $[13,14]$. Several issues can now be addressed: the shape and temperature dependence of the velocity curves (Fig. 1), but also the
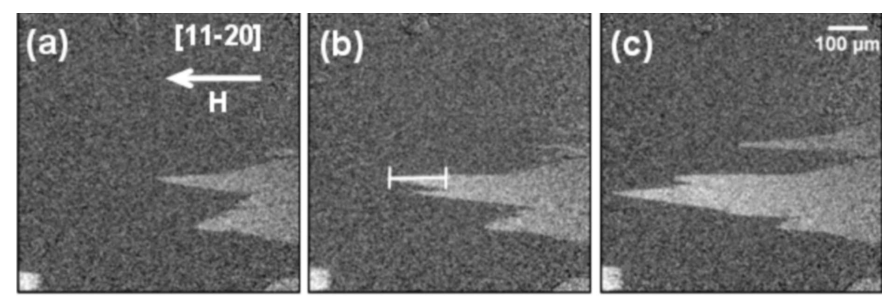

FIG. 3. Kerr microscopy images of magnetic domains taken at $\mathrm{T}=270 \mathrm{~K}$ after 3 successive propagation pulses of amplitude $4.1 \mathrm{mT}$ and duration $0.6 \mu \mathrm{s}$. 
possible mechanisms at play in the DW propagation in the coexistence regime.

The 1D DW propagation model is a good starting point to explain these curves. Initially developed to describe the propagation of Bloch DWs in perpendicularly magnetized layers [23, 24], it was later adapted to head-tohead or tail-to-tail charged walls in in-plane magnetized tracks [25]. This is the situation encountered at the apex of the domain tips in MnAs. When compared to micromagnetic simulation results, the 1D model was found to provide the correct behavior of velocity-versus-field curves although the maximum velocity could differ by a factor of two. DW propagation exhibits features similar to the perpendicular case: a stationary regime at low field, followed by a precessional regime beyond the Walker field $\mathrm{H}_{W}$. The model assumes effective anisotropy constants with an anisotropy energy of the form: $E_{\text {anis }}=$
$K_{0} \sin ^{2} \theta+\sum_{n=1}^{\infty} K_{n} \sin ^{2 n} \theta \sin ^{2}\left(n \Phi+\Phi_{n}\right)$, where $\theta$ is the polar angle of the magnetization vector with respect to the in-plane easy axis $(x)$ along which the DW propagates and $\Phi$ the azimuthal angle. Neglecting the long-range contribution of the charged wall to the effective magnetic anisotropy and taking into account the symmetry of the magnetic anisotropy in MnAs we keep only the $K_{1}$ term in the summation and use the following relations: $K_{0}=K_{2 \|}-K_{2 \perp}+\mu_{0} M_{s}^{2} / 2$ and $K_{1}=K_{2 \perp}-\mu_{0} M_{s}^{2} / 2$ where $M_{s}$ is the saturation magnetization. Assuming that the DW position can be fully described by the sole time derivative of the angle $\theta$ (1D approximation), and using the propagation equations thus derived from the Landau-Lifshitz-Gilbert equation, the DW velocities in the stationary and precessional regime are given by:

$$
v_{\text {stat }}=\frac{\gamma \Delta_{0} \mu_{0} H}{\alpha}\left(1+\frac{\kappa}{2}\left(1-\sqrt{1-\left(\frac{H}{H_{W}}\right)^{2}}\right)\right)^{-1 / 2},\left\langle v_{\text {prec }}\right\rangle=\frac{1}{T} \int_{0}^{T} \Delta(t)\left[\alpha \frac{d \Phi}{d t}+\frac{\gamma \mu_{0} H_{W}}{\alpha} \sin (2 \Phi)\right] d t,
$$

with the DW width $\Delta=\Delta_{0} / \sqrt{\left(1+\kappa \sin ^{2} \Phi\right)}$, $\Delta_{0}=\sqrt{A / K_{0}}$ and the time-dependence of the azimuthal angle given by $\tan \Phi=\frac{H_{W}}{H}+$ $\sqrt{1-\left(\frac{H_{W}}{H}\right)^{2}} \tan \left[\frac{\gamma \mu_{0} H_{W}}{1+\alpha^{2}} \sqrt{\left(\frac{H_{W}}{H}\right)^{2}-1} t\right] . \quad \alpha \quad$ is the Gilbert damping factor, $\kappa=K_{1} / K_{0}$ and $H_{W}$ $=\alpha K_{1} / \mu_{0} M_{s}$. Figure 4 shows the velocity curve calculated from Eq. 1 with the micromagnetic parameters ( $K_{1}, K_{0}, A$ and $M_{s}$ ) obtained experimentally (full curve). The velocity rises with a sublinear behavior (stationary regime) up to a field very close to the Walker field. After that it decreases since the DW enters the precessional regime where it moves back and forth, hence with a lower average velocity. Actually the shape of the velocity curve, especially in the stationary regime, depends mostly on the value of $\kappa[22,25]$, equal to 1.1 here. The maximum velocity is proportional to $\Delta_{0}$.

The experimental curves can then be qualitatively understood as shown in Fig. 4 for the $\mathrm{T}=290 \mathrm{~K}$ curve. The depinning regime starts at $H_{d e p}$. The velocity rises but with an average value lower than in the intrinsic stationary regime since the wall is pinned part of the time. The end of the depinning regime occurs at the field $H_{f l}$ where the curve meets the intrinsic flow regime. If $H_{f l} \geq H_{W}$ the velocity curve shows a peak at $H_{f l}$. This scenario qualitatively explains the experimental velocity curve at $290 \mathrm{~K}$ provided the Gilbert damping factor is taken equal to 0.028 . This value is larger than the one we determined by FMR (homogeneous damping $\alpha=0.002$ for a 15 $\mathrm{nm}$ thick layer) as usually encountered in DW propaga- tion experiments (see Ref. [26] and references therein), but compatible with the one extracted from the pumpprobe experiments in Ref. [27] $(\alpha=0.017)$. The $K_{0}$ constant is also in principle an adjustable parameter since it should include phenomenologically the contribution of the demagnetization energy from the charged wall (demagnetization field along the easy axis, opposite to the $x$-component of the magnetization). The dashed curve in Fig. 4, which is in very good agreement with the experimental one, is calculated using $K_{0}=0.810^{5} \mathrm{~J} \mathrm{~m}^{-3}$. Simulations for the $200 \mathrm{~K}$ and $270 \mathrm{~K}$ curves using the anisotropy constants of Refs. [18, 19] and estimated exchange constants show that the succession of dynamical regimes is likely to be the same, i.e. first a depinning regime up to the maximum velocity and then the precessional flow regime. Let us note that $H_{f l}$ is larger by a factor of 2 at $290 \mathrm{~K}$ as compared to $200-270 \mathrm{~K}$. This might indicate that the Walker field is larger at $290 \mathrm{~K}$ presumably because of the decrease of $K_{1} / M_{s}$ with temperature.

At $290 \mathrm{~K}$ the sample is in the early stages of the transformation of the $\alpha$ phase into a mixed phase, which raises the question of the effect of the $\beta$ phase on the expansion of the domains. The magnetic domain pattern observed at this temperature is unchanged from the one shown in Fig. 3, in particular the angle of the wedged domains has not varied: the displacement of the side walls of domains appears not to have been much disturbed. This is consistent with the $\mathrm{T}=16^{\circ}(\mathrm{T}=289 \mathrm{~K})$ XMCD-PEEM images in Ref. [28] showing a very dilute $\beta$ phase, and 


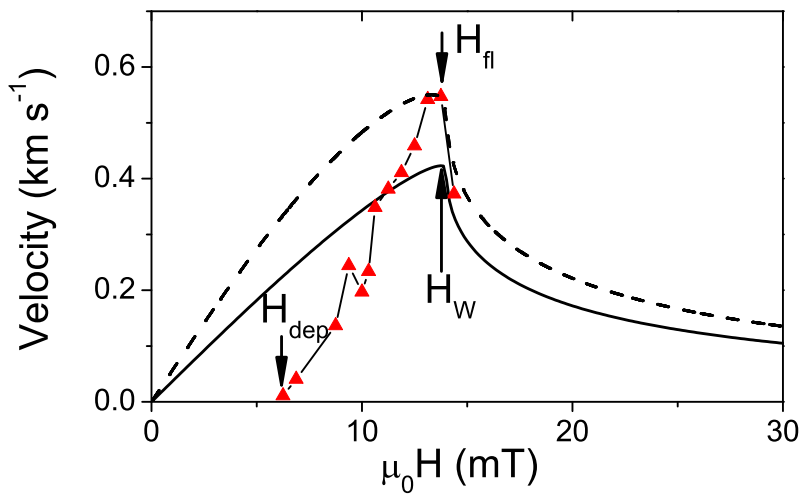

FIG. 4. DW velocity calculated from Eq. (1) with $A=6.6 \mathrm{pJ} \mathrm{m}^{-1}, K_{1}=2.110^{5} \mathrm{~J} \mathrm{~m}^{-3}, K_{0}=1.910^{5} \mathrm{~J} \mathrm{~m}^{-3}$ (full curve) and $K_{0}=0.810^{5} \mathrm{~J} \mathrm{~m}^{-3}$ (dashed curve), $\alpha=0.028$. Symbols: experimental curve at $\mathrm{T}=290 \mathrm{~K}$.

the persistence of the pointed domains, unhindered by the nucleating stripes. The main effect of the coexistence of the $\alpha / \beta$ phase on the DW velocity at $\mathrm{T}=290 \mathrm{~K}$ is found in a depinning regime that extends from 6 to $13.5 \mathrm{mT}$, i.e. over a larger range than at lower temperatures. In the flow regime however, the DWs easily go past the defects formed by the nucleating $\beta$ phase, and the velocity reached at $\mathrm{H}_{f l}$ is high, in agreement with the simple 1D model. A more elaborate model taking into account the full structure of the DW would likely refine the predicted velocities. Above $\mathrm{T}=290 \mathrm{~K}$, the loss of Kerr contrast is very likely due to the domains being broken up by the non-ferromagnetic stripes whose volume fraction increases rapidly with temperature.

As a conclusion we have studied the field induced DW dynamics in a MnAs/GaAs (100) film and identified distinct depinning and precessional flow propagation regimes. We highlight here that the early stage of the $\alpha-\beta$ transformation in MnAs offers the unique opportunity to study the effect of a temperature-dependent and totally reversible well-defined DW pinning potential landscape. The high velocity reported near room temperature makes MnAs a promising candidate for magneto-logic devices, which would require nano-patterning. From this study, we can anticipate that nanostructuring these films into wires parallel to the easy axis would give similar results, i.e high DW velocities relatively unhindered by the $\beta$ phase at $290 \mathrm{~K}$. In this geometry, the DW will be charged and wide, reduced to the apex part of the domains in this study. Patterning wires along the hard axis has already shown that the organization of the mixed phase notably changes [16]. Under applied field, one further expects the propagation of pure, uncharged Bloch walls propagating along the structure. As these are narrower, DW velocities in these structures can be expected to be smaller than when patterned along the easy axis.

We acknowledge valuable technical assistance from $\mathrm{M}$. Bernard and S. Majrab at INSP.
[1] S. S. P. Parkin, M. Hayashi, and L. Thomas, Science 320, 190 (2008).

[2] H. T. Zeng, D. Read, L. O'Brien, J. Sampaio, E. R. Lewis, D. Petit, and R. P. Cowburn, Appl. Phys. Lett 96, 262510 (2010).

[3] G. S. D. Beach, C. Knutson, C. Nistor, M. Tsoi, and J. L. Erskine, Phys. Rev. Lett 97, 057203 (2006).

[4] M. Hayashi, L. Thomas, C. Rettner, R. Moriya and S.S.P. Parkin, Nat. Phys. 3, 21 (2007).

[5] M. Tanaka, Semicond. Sci. Technol. 17, 327 (2002).

[6] S. H. Chun, S. J. Potashnick, K. C. Khu, P. Schiffer, and N. Samarth, Phys. Rev. B 66, 100408(R) (2002).

[7] V. Garcia, H. Jaffres, M. Eddrief, M. Marangolo, V. H. Etgens and J. M. George, Phys. Rev. B 72, 081303(R) (2005).

[8] M. Tanaka, J. P. Harbison, M. C. Park, Y. S. Park, T. Shin, and G. M. Rothberg, Appl. Phys. Lett. 65, 1964 (2008).

[9] N. Mattoso, M. Eddrief, J. Varalda, A. Ouerghi, D. Demaille, V. H. Etgens, and Y. Garreau, Phys. Rev. B 70, 115324 (2004).

[10] P. J. Metaxas, J. P. Jamet, A. Mougin, M. Cormier, J. Ferré, V. Baltz, B. Rodmacq, B. Dieny and R. L. Stamps, Phys. Rev. Lett. 99, 217208 (2007)

[11] A. Dourlat, V. Jeudy, A. Lemaître, and C. Gourdon, Phys. Rev. B 78, 161303 (2008).

[12] T. Manago, H. Akinaga, Surface Science 600, 3492 (2006)

[13] K. S. Ryu, S. C. Shin, H. Akinaga and T. Manago, Appl. Phys. Lett 88, 122509 (2006).

[14] K. S. Ryu, H. Akinaga, and C. S. Shin, Nat. Phys. 3, 547 (2007).

[15] S. C. Shin, K. S. Ryu, D. H. Kim, and H. Akinaga, J. Appl. Phys. 103, 07D907 (2008).

[16] M. Tortarolo, M. Sirena, J. Milano, L. B. Steren, F. Vidal, B. Rache Salles, V. H. Etgens, M. Eddrief, G. Faini, and L. I. Pietrasanta, Phys. Rev. B 81, 224406 (2010).

[17] F. Schippan, G. Behme, L. Daweritz, K.H. Ploog, B. Dennis, K. U. Neumann and K. R. A. Ziebeck, J. Appl. Phys. 88, 2766 (2000).

[18] L. B. Steren, J. Milano, V. Garcia, M. Marangolo, M. Eddrief, and V. H. Etgens Phys. Rev. B 74, 144402 (2006).

[19] J. Lindner, T. Toliński, K. Lenz, E. Kosubek, H. Wende, K. Baberschke,A. Neyb, T. Hesjedal, C. Pampuch, R. Koch, L. Däweritz, K.H. Ploog, J. Mag. Magn. Mat. 277, 159 (2004).

[20] Our value of the exchange constant $A$ is about 5000 times larger than the one determined in T. Toliński, J. Lindner, K. Lenz, K. Baberschke, A. Ney, T. Hesjedal, C. Pampuch, L. Daweritz, R. Koch and K. Ploog, Europhys. Lett. 68, 726 (2004) ( $A=1.7710^{-14} \mathrm{~J} \mathrm{~m}^{-1}$ at room temperature). There is most probably an error by a factor 1000 in the determination of $A$ in this work (field in kOe instead of Oe).

[21] A. Hubert and R. Schäfer, in Magnetic Domains, Springer (Berlin) 2000.

[22] L. Thevenard, S. A. Hussain, H. J. von Bardeleben, M. Bernard, A. Lemaître, and C. Gourdon, Phys. Rev. B 85, 064419 (2012).

[23] J. C. Slonczewski, J. Appl. Phys. 44, 1759 (1973).

[24] N. L. Schryer and L. R. Walker, J. Appl. Phys. 45, 5406 
(1974)

[25] A. Thiaville and Y. Nakatani, in Spin dynamics in confined magnetic structures III, ed. by B. Hillebrand and A. Thiaville, Topics Appl. Physics 101, p. 161, Springer (Berlin) 2006.

[26] A. Dourlat, V. Jeudy, A. Lemaître and C. Gourdon, Phys. Rev. B 78, 161303(R) (2008).

[27] K.-D. Lee, K.-S. Ryu, J.-W. Kim, H.-S. Song, J.-W.
Jeong, and S.-C. Shin, Phys. Rev. B 82140401 (2010).

[28] R. Engel-Herbert, A. Locatelli, S. Cherifi, D. M. Schaadt, J. Mohanty, K.H. Ploog, E. Bauer, R. Belkhou, S. Heun, A. Pavlovska, T. Leo, T. Hesjedal, Appl. Phys. A 84, 231 (2006).

[29] V. M. Kaganer, B. Jenichen, F. Schippan, W. Braun, L. Däweritz, and K. H. Ploog, Phys. Rev. B 66,045305 (2002). 